\title{
Pose-invariant Face Recognition by Matching on Multi-resolution MRFs linked by Supercoupling Transform
}

\author{
Shervin Rahimzadeh Arashloo, Josef Kittler and William J. Christmas \\ Center for Vision, Speech and Signal Processing \\ University of Surrey \\ Guildford GU2 7XH, United Kingdom
}

\begin{abstract}
Recognition of faces in arbitrary pose is addressed in this paper. For this task, an MRF-based classification approach is proposed which employs the energy of the established match between a pair of images as a criterion of goodness-of-match. By incorporating an image matching method as part of the recognition process, the system is made robust to moderate global spatial transformations. The approach draws on the method in [1] which has the potential to cope with pose changes but a direct application of which suffers from several shortcomings. In order to overcome these problems, a number of enhancements are proposed. First, by adopting a multi-scale relaxation scheme based on super coupling transform, the inference using sequential tree re-weighted message passing approach [2] is accelerated. Next, by taking advantage of a statistical shape prior for the matching, the results are regularized and constrained, making the system robust to spurious structures and outliers. For classification, both textural and structural similarities of the facial images are taken into account. The method is evaluated on two databases and promising results are obtained.
\end{abstract}

Keywords: Face recognition, pose-invariance, image matching, Multi-resolution MRfs, Super-coupling transform

\section{Introduction}

In spite of the recent advances in face recognition, recognizing faces across different poses still remains a major challenge [3]. The performance of face 
recognition algorithms is seriously affected by any deviation of the acquired image pose from frontal. The importance of the problem is evident from the variety of applications in which no control can be imposed on the imaging direction. These include security and surveillance scenarios, online image search and tagging, analysis of personal photos, etc. The difficulty of the problem stems from the fact that in a classification system, very often the between-class scatter of data is dominated by the within-class variance attributed to pose changes which results in a loss of discriminative identity information.

It is known that in the presence of inconsistencies in the imaging situations between the gallery and test images (e.g. pose changes), holistic approaches for object recognition do not perform well. In these cases, a well known alternative which has also been successfully applied to other object recognition problems [4], is based on the premise that concepts of interest are constructed from distinctive configurations of symbolic interconnecting primitives. In this context, undirected graphical models, commonly referred to as Markov random fields (MRFs) have extensively been used as a suitable framework for representing image content as well as for modeling. Accordingly, the primitives are represented as nodes of the graph while edges (hyperedges) encode their interaction and conditional dependence/independence properties. For classification, an unknown pattern is matched to the prototypes of all classes with respect to the contextual relationships of its components and is assigned to the class exhibiting maximum similarity. In a recognition scenario, the quality of a match is often evaluated in terms of the maximum a posteriori probability of the corresponding configuration of the Markov random field (MAP-MRF), or inversely the posterior energy of the established match is taken as the cost of matching. The problem then becomes one of finding the mode of a distribution which is factorized over the graph.

The current work applies this general concept to recognition of faces under arbitrary pose where only frontal images are available as class exemplars.

\section{Overview}

A test image which can potentially be captured under a non-frontal pose, is matched to the prototypes of all classes (which are in frontal pose) and classified according to both its textural and structural characteristics.

For matching, we adopt an efficient deformable image matching method proposed in [1] and adapt it to our framework. The general ideas employed 
here appeared also in a preliminary version of the current work [5]. The modifications to the matching method in [1] and to the method in [5] include: a multi-resolution approach based on the supercoupling transform to establish pixel-wise correspondences while keeping the computational cost to a minimum and regularizing and constraining the solution by incorporating a statistical shape prior in the matching. Also, although in [5] we did not align test images using eye coordinates, we used them to crop the faces out of the image. In contrast here we make use of the face detection method in [6] without using eye coordinates.

Once pixel-wise correspondences have been established between visible parts of a pair of images, the textures are characterized using Uniform Local Binary Pattern Histograms (ULBPH) while the useful discriminatory shape differences (local deformations) are measured in the shape space constructed by PCA. A nearest neighbor classifier designed in the space of shape and texture similarities is then employed to classify the unknown pattern.

In comparison to the existing approaches for handling the pose variation problem in face recognition, some of the distinctive attributes of the proposed method can be summarized as follows:

- no need to detect landmark points prior to recognition,

- no need to train on non-frontal images,

- low computational complexity compared with 3D-based approaches,

- and no need for prior information about the pose of the subject,

A few comments regarding the use of landmark points are in order. We use the provided eye coordinates for cutting the face out of the image for frontal gallery images only. For the test images we use the automatic method in [6] to detect a face in the image. As a result, not only the test images are not aligned to the gallery images and might be in different scales, there might be some errors in detection which adversely affect the performance. In comparison to the existing face recognition algorithms, the proposed method resembles more a real face recognition system since automatic detection of eyes for an arbitrary pose is a difficult task. The situation becomes even worse when the subject blinks.

The paper is organized as follows. In Section 3, we review the literature on pose-invariant face recognition methods. In Section 4, the image matching method in [1] is overviewed. In Section 5, the proposed multiresolution 
relaxation based on the supercoupling transform is described. Constructing and employing a PCA-based shape prior for regularizing and constraining the solution is presented in Section 6. In Section 7, after describing a classification scheme, which takes advantage of both texture and shape similarities of faces, the experimental setup and the test results are discussed. In Section 9 , conclusions are drawn.

\section{Related Work}

Among the early attempts to generalize face recognition algorithms so that they become applicable across different poses are direct extensions of those developed for frontal images. Such algorithms require a large number of training data to cover the viewing sphere [7, 8]. More recent attempts trying to take advantage of multiple gallery images of different poses include the work in [9] in which the goal is to avoid the complexity of estimating the 3D structures of images and to obviate the need for the storage of multiple templates of each subject. A closely related approach is to use multiple images at some specific views and build a model which can generate novel views [10] by interpolation.

A second group of methods use only a single gallery image per subject, although for training, multiple images captured under different poses or under different illumination conditions might be required. These approaches can be further classified into two distinguishable subsets. In the first set which can be considered as generative methods, virtual views in the desired poses are synthesized using either 2D learning-based methods or 3D model-based approaches. In the second class one seeks to find an optimal decision rule for better discrimination between classes without generating any novel views. As an example of the 2D learning-based methods for virtual view synthesis is the work in [11] in which using multiple 2D images of each subject under different illumination conditions, 3D shape and albedo of the faces are estimated and then used for generating virtual views. In [12], based on the assumption that a 3D shape can be represented as a linear combination of prototypical views of an object in $2 \mathrm{D}$, the authors extended their previous work in [8] by generating virtual views using prior knowledge of shape and texture of faces in 2D and then employed a view-based face recognizer. By investigating point distribution models and the eigenvectors responsible for pose changes, in [13], the authors created virtual views. Another well known example is based on the Active Appearance Model which uses statistical models of shape 
and texture for constructing a 2D face model [14]. One of the shortcomings of this approach is that as soon as in-depth rotation of head becomes large, the assumptions governing the model collapse and the model fails to work properly. In order to handle larger rotation angles, in [15] a number of models trained for different view points are used. As a different approach, designed to facilitate an effective use of mugshot databases, a method is proposed in [16] to generate virtual views. Using frontal and side-view face images and a number of manually labeled landmark points, the 3D shape and texture of the subject are estimated. For recognition, appearance based face recognition methods are employed. In [17], a bayesian generative model is proposed to transform features from the identity space into the observation space using a deterministic function. Several local models describing the change of each local feature are involved. The parameters of the transformation functions are learned using the EM algorithm, assuming that the pose is known a priori.

In [18], eigenlight-fields are used to tackle the pose variation problem. Single gallery and probe images are assumed to be drawn from a larger data pool containing images of different poses and illumination conditions. Recognition is formulated as a missing data problem. Using the prior knowledge of the distribution of the complete gallery set, the missing information is inferred and a few eigenvectors are used for recognition.

A commonly adopted method in the context of pose-invariant face recognition is to employ $3 \mathrm{D}$ models, the most well known one in this category being the 3D morphable model [19]. These methods do not need multiple images from different view points. Instead, 3D (laser-scanned) data is needed for training, although these do not have to be of the same identities as those of the gallery images. In these techniques, statistical models for shape and texture are constructed offline. For a new image, after fitting the model, shape and texture parameters are recovered. For recognition, two different approaches can be considered. One may directly use the derived parameters of the model for recognition, as in e.g. [20, 21, 22]. The second approach is to apply a geometric normalization step and render the probe image in a desired pose (usually frontal) and then use 2D-based methods for recognition [23]. Some of the drawbacks of these methods are as follows. The most important disadvantage is that the recovered shape and texture in 3D geometric normalization based approaches are completely affected by the model which has the capacity to reconstruct only the information captured during statistical learning. As a result, these techniques are not able to recover ir- 
regular features that have not been available in the training set. Moreover, the high computational burden of the 3D methods disqualifies them for realtime applications. Finally, for fitting the model, at least six landmark points are needed which are provided manually.

The second subset of methods which use a single gallery image for each subject are the discriminative approaches. In comparison to the first group, in these approaches no virtual views are generated. Instead, one tries to extract and use features that lie in a pose-invariant space in which the identity is assigned based on the distance in that space. As an example, in [24], a locally linear discriminant analysis (LLDA) approach is employed to model nonlinearities of the face data manifold and to extract class-discriminative features. In [25], the authors propose to use a kernel-based LDA approach. A method is proposed for automatically tuning the parameters of the kernel function in a kernel-based LDA method. The authors attempt to improve the generalization capability of kernel-based LDA by maximizing the margin criterion while retaining the stability of the method.

In [26], the authors propose a probabilistic approach to handle changes in the viewing angle by formulating the similarity of an image pair as the sum of similarities between different subregions. In order to take into account the change in appearance of different regions due to pose variations, images of different poses are used to learn the distribution of region similarities across different poses. In [27], after dividing the images into non-overlapping patches, the authors try to learn their deformation and appearance as a result of pose changes. The similarity of patches is measured in terms of their gray levels, and the overall similarity of two faces is defined as a probabilistic measure of local patch similarities without any attempt to use shape information explicitly.

A relatively different approach to tackle the pose variation problem is based on the idea that 2D projections into an eigenspace of a $3 \mathrm{D}$ object viewed from different angles, fall on a 2D manifold [28]. As an example of the works in the literature which make use of dense correspondences between images for recognition, is the work in [29] which uses stereo matching for recognition. The error brought about by the hypothesis that the pixels have only 1D disparities is problematic when a change of viewing angle results in 2D disparities even after aligning the two images using the eye coordinates. The similarity measure used in [29] is based on the gray scale content of images only, ignoring the geometric distortion which can be informative for recognition. 
There are similar approaches in the literature which are based on the idea of MRFs and graphical models. Although in these works no attempt is made to recognize faces across different poses, we briefly review some of them because of their similarities to the current approach. In [30], face recognition is formulated as a bayesian decision making problem based on MRFs. Local features are acquired using Gabor filters and contextual relationships between sites are modeled by a linear penalty function. The final similarity measure between two faces is defined as the matching cost. In [31], a face is represented by a face-ARG model. Straight line segments are extracted and used as face features, i.e. nodes of the graph. Adding proper binary relations between nodes, a partial matching is used to match different pairs of graphs and to select the best match for recognition. In [32], the authors employ an MRFbased method in which individual sites are local patches of images. For recognition, each patch is assigned to one possible class of identities using belief propagation for inference, and for classification, a majority vote rule is employed.

Despite the capacity of MRFs in image modeling and also the emergence of successful inference methods on them, application of these models in the context of pose-invariant face recognition has been very limited. In the current work, by taking advantage of an image matching method based on MRFs and a successful inference scheme, the similarity between a pair of images is gauged as the energy of the match and then used for hypothesis selection.

\section{Image Matching}

In the following we describe a matching method based on graphical models. In the passing we note that there are other non-MRF based methods for image matching and alignment such as those in [33, 34, 35].

As noted earlier, in modeling images using graphical models, individual features are symbolized as nodes of the graph while edges encode conditional dependencies and the neighborhood structure. The goal is then to assign each node a class from a predefined admissible discrete set. Let $\mathcal{V}=\{1, \ldots, m\}$ denote a set of nodes and $L=\{1, \ldots, K\}$ a discrete set of labels. The labeling problem is defined as one of assigning a label from set $L$ to each site of $\mathcal{V}$ subject to contextual constraints in a predefined neighborhood system. A random field $F=\left\{F_{1}, \ldots, F_{m}\right\}$ is a group of random variables defined on the set $\mathcal{V}$ in which a value $x_{s}$ in $L$ is assigned to each variable $F_{s}$. Denoting the event that $F_{s}$ takes value $x_{s}$ by $F_{s}=x_{s}$ and the joint event by $\left(F_{1}=\right.$ 
$\left.x_{1}, \ldots, F_{m}=x_{m}\right)$, then $X=\left\{x_{1}, \ldots, x_{m}\right\}$ is called a configuration of $F$. If we denote the joint probability of the random variable $F$ taking value $X$ by $P(X)$, then $F$ is called a Markov random field on $\mathcal{V}$ with respect to a neighborhood system $N$ if and only if the positivity and Markovianity conditions are satisfied:

$$
\begin{array}{cl}
\text { Positivity } & : P(X)>0, \forall X \in \mathcal{F} \\
\text { Markovianity } & : P\left(x_{s} \mid x_{\mathcal{V}-\{s\}}\right)=P\left(x_{s} \mid x_{N_{s}}\right)
\end{array}
$$

where $\mathcal{V}-\{s\}$ stands for the difference of two sets, $x_{\mathcal{V}-\{s\}}$ is the set of labels at the sites in $\mathcal{V}-\{s\}$ and $x_{N_{s}}$ is the set of labels of the neighboring sites of $s$ :

$$
x_{N_{s}}=\left\{x_{s^{\prime}} \mid s^{\prime} \in N_{s}\right\}
$$

and $N_{s}$ is the neighborhood of site $s$. When only cliques of size up to two are considered, the posterior energy, considered as the minus log probability of the configuration, has the following form:

$$
E(X \mid \theta)=\sum_{s \in \mathcal{V}} \theta_{s}\left(x_{s}\right)+\sum_{(s, t) \in \mathcal{E}} \theta_{s t}\left(x_{s}, x_{t}\right)
$$

$\mathcal{V}$ is the set of sites and $\mathcal{E}$ represents the set of edges. $\theta$ parameterizes the energy and is dependent on the application in hand.

The image matching problem can be posed as a labeling problem on MRFs. Labels in this case correspond to two dimensional displacement vectors. In a naive realization of the problem, the label set is expressed as the Cartesian product of two sets, each of which represents the admissible disparities in one direction:

$$
L=\left\{\left(x_{s^{1}}, x_{s^{2}}\right) \mid\left(x_{s^{1}}, x_{s^{2}}\right) \in L_{s^{1}} \times L_{s^{2}}\right\}
$$

where $L_{s^{1}}$ and $L_{s^{2}}$ stand for the admissible labels in each direction and $x_{s^{1}}$ and $x_{s^{2}}$ denote displacements (labels) of site $s$ in two directions. Using this simple realization of matching, the task becomes intractable as soon as disparities in each direction grow. Instead of the product model, the work in [1] formulates the image matching as a labeling problem on two MRFs interacting together. The label set in this case is defined as:

$$
L=\left\{\left(x_{s^{1}}, x_{s^{2}}\right) \mid x_{s^{1}} \in L_{s^{1}}, x_{s^{2}} \in L_{s^{2}}\right\}
$$




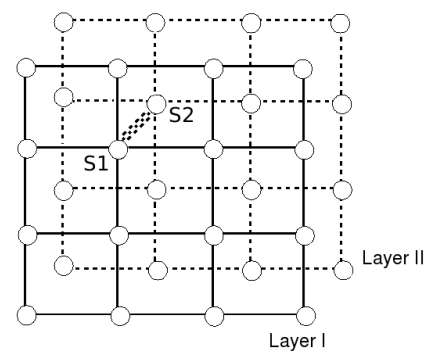

Figure 1: Two MRFs interacting together with a sample inter-layer edge between them.

The efficiency of this method takes on the idea of decomposition of the 2D label set into two 1D distinct label sets, each representing the label set for one MRF. The edge set of this model is comprised of two separate edge sets (inter-layer and intra-layer edges). The edge potential functions on each of these layers are assumed to be identical (intra-layer edges) while inter-layer edges encode the data term. For the intra-layer edges, the following crisp continuity terms are used:

$$
\theta_{s t}\left(x_{s}, x_{t}\right)= \begin{cases}0, & x_{s}=x_{t} \\ c_{r}, & \left|x_{s}-x_{t}\right|=1 \\ \infty, & \left|x_{s}-x_{t}\right|>1\end{cases}
$$

The inter-layer edges encode the data term, i.e. the cost of assigning label $x_{s^{1}}$ in layer one and label $x_{s^{2}}$ in layer two to two isomorphic nodes of the graph. The data term has been constructed using the block model in which pixels are grouped into non-overlapping blocks. Assuming that the target image is a noisy observation of the template image and assuming a guassian distribution with zero mean and variance of $\sigma^{2}$ for the noise, the data term for the block model is defined as:

$$
\theta_{s^{1} s^{2}}\left(x_{s^{1}}, x_{s^{2}}\right)=\frac{1}{2 \sigma^{2}} \operatorname{Dis}\left(I_{\vec{s}}^{1}, I_{\vec{s}+\left(x_{s^{1}}, x_{s^{2}}\right)}^{2}\right), s^{1} \in \mathcal{V}^{1}, s^{2} \in \mathcal{V}^{2}
$$

where $I_{\vec{s}}^{1}$ is a block with the coordinates $\vec{s}$ on image $I^{1}$ and the corresponding block with the coordinates $\vec{s}+\left(x_{s^{1}}, x_{s^{2}}\right)$ on image $I^{2}$ is denoted by $I_{\vec{s}+\left(x_{s^{1}}, x_{s^{2}}\right)}^{2}$. $\vec{s}$ denotes the vector pointing to the spatial position of block $I_{\vec{s}}^{1}$. $\operatorname{Dis}(.,$.$) is$ a dissimilarity measure which is defined as the sum of squared differences of colors over the pixels of corresponding blocks. Figure 1 illustrates the two 
MRFs interacting together with a sample inter-layer edge encoding the data term.

In order to achieve more flexibility in deformation, in the definition of pairwise potentials we replace hard continuity terms by a quadratic penalty function:

$$
\theta_{s t}\left(x_{s}, x_{t}\right)=\rho\left(x_{s}-x_{t}\right)^{2}
$$

where $\rho$ is a normalizing constant. The value of constant term $\rho$ depends on the range of input data (scaled to $[-1,1]$ in our case) and also controls the elasticity of the model.

For the computation of the data term, we use edge maps since they are less affected by unwanted illuminations changes. Horizontal and vertical edges are scaled to the range $[-1,1]$ and combined to form the data term. The data term is defined as:

$$
\begin{array}{r}
\theta_{s^{1} s^{2}}\left(x_{s^{1}}, x_{s^{2}}\right)=\frac{1}{2 \sigma^{2}}\left[\operatorname{Dis}\left(I_{\vec{s}}^{1 h}, I_{\vec{s}+\left(x_{s^{1}}, x_{s^{2}}\right)}^{2 h}\right)+\operatorname{Dis}\left(I_{\vec{s}}^{1 v}, I_{\vec{s}+\left(x_{s^{1}}, x_{s^{2}}\right)}^{2 v}\right)\right], \\
s^{1} \in \mathcal{V}^{1}, s^{2} \in \mathcal{V}^{2}
\end{array}
$$

where $I_{\vec{s}}^{1 h}$ and $I_{\vec{s}+\left(x_{s}, x_{s}\right)^{2}}^{2 h}$ denote a block in the horizontal edge map of the first image and its corresponding block in the horizontal edge map of the target image, respectively. $I_{\vec{s}}^{1 v}$ and $I_{\vec{s}+\left(x_{s} x^{1} x_{s}\right)}^{2 v}$ are defined in a similar way.

In order to rely more on the common features of the two images and bypass the atypical features which exist only in one image, weak edges are ignored and at the same time the data term is truncated. By truncating the data term, the matching becomes more robust to outliers, occlusions or features which appear only in one image.

The main modifications to the method in [1] can be outlined as follows:

- In [1] pixels are grouped into blocks to reduce the computational cost, this compromises the accuracy of matching by inferring one motion vector for each block instead of individual pixels. In contrast we circumvent this shortcoming by employing a multi-resolution approach going down to the pixel level for matching while keeping the computational cost to a minimum.

- The pairwise potentials in the method in [1] only impose a general smoothness prior and are not object specific. It is naturally expected that incorporating a shape prior would improve the results. In order 
to constrain the solution, we take advantage of a PCA-based shape prior. Since the inference using higher order clique introduced by the prior term is very inefficient, we follow a two-stage process in order to incorporate the prior knowledge of the object shape into the inference.

\section{Multi-scale Relaxation}

A common framework for inference on a graph is the decomposition approach. The idea is to decompose the original loopy graph into smaller subproblems for each of which exact inference is realizable. Two famous examples in this category are the TRW-S [2] and max-sum diffusion [36]. Both methods solve the original problem by considering smaller subproblems. While the subtasks in max-sum diffusion are individual edges, in TRW-S with monotonic chains, smaller subproblems are assumed to be horizontal and vertical trees in each row and column of the image. TRW-S achieves faster convergence by employing larger subproblem (trees vs. individual edges). Solving the inference on each individual tree is then realized using the maxproduct algorithm which employs localized computations according to the general distributive law [37] to reduce computational burden. Further information about the comparison between different inference schemes can be found in [38]. The computation of messages, a time consuming part in the TRW-S (and all other message passing approaches) can be performed using distance transform [39]. As noted earlier the method in [1] takes advantage of the node decomposition approach and the block model for speed up. While the method is made more robust against noise by using block model, inferring a single motion vector for every 16 pixels reduces the accuracy of the established match. Alternatively one can build the model considering each individual pixel of the image as a node. Unfortunately this approach increases the computational cost prohibitively and the optimization would be prone to local minima. Multiresolution analysis has been successfully employed for avoiding these problems.

One of the common approaches in multiresolution optimization is based on the Renormalization Group Transform (RGT) [40, 41]. This approach is known to preserve the whole structure of the probability distribution. However, in most cases, one is not interested in preserving the full structure of the probability distribution, but only in preserving its maxima (just as in our task). In these cases, a potential-based coarsening technique (supercoupling transform [42]) which is known to be order preserving, in the sense that the 
inequalities obeyed by the original distribution remain true after coarsening is employed. A natural consequence of order preserving property is that the mode of the original distribution maps exactly to the mode of the coarsened distribution. In fact, the multiresolution approach we employ here is intended to accelerate the optimization process by coarsening the configuration space so that long range jumps that would lead faster to the global maximum are possible. Other potential benefits of the multiresolution approach applied here can be considered as reducing the duality gap in difficult problems, reducing the number of message passing operations and as a result achieving accelerated convergence.

One of the considerations in multiresolution analysis is the transformation of the solution obtained in one level to a finer level. According to the theory of RGT this solution should be used to restrict the solution in the finer level and only those configurations which are consistent with the coarser level should be considered. A common practice is to use a block-flat assumption and assign all nodes inside a block the same label. In this way, the solution obtained in the coarser level serves as a starting point for the optimization in the finer level. Under the block-flat assumption, the supercoupling transform requires that the value of the cost function when going from one level of resolution to another should remain the same. It is shown that this transformation at the zero temperature limit is identically the same as RGT [42]. There are some other multiscale approaches such as $[43,44]$ in the literature. The technique employed here applies multiresolution ideas in a principled way, based on supercoupling transform. Also, in the core of the method, TRW-S is used which enjoys better convergence properties than belief propagation used in other works.

\subsection{The Optimization Process}

For brevity we will consider only two levels of resolution referring to them as the coarse and the fine level. We assume that images are of size $2^{n} \times 2^{n}$. We coarsen the image lattice by replacing every four nodes by one node in the coarse lattice. Thus, each node in the coarse lattice (denoted by $\mathrm{s}$ ) corresponds to four nodes in the finer lattice (denoted by $s_{1}, s_{2}, s_{3}$ and $s_{4}$ ) as illustrated in Fig. 2. We use the symbol $\bar{X}$ to denote the fine configuration that can be produced under the block-flat assumption from the coarse configuration $X^{\prime}$. The theory of supercoupling transform then says that the parameters of the energy should be chosen in such a way that

$$
E(\bar{X})=E\left(X^{\prime}\right) .
$$




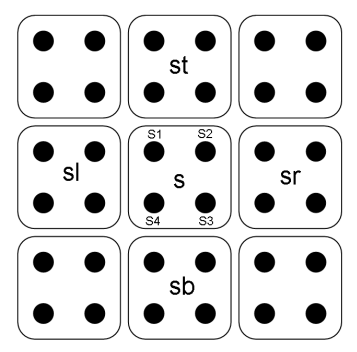

Figure 2: Geometry of sites in the coarse and fine lattice under consideration.

For each site in the coarse lattice and its four corresponding sites in the fine level the following equation must hold:

$$
\begin{array}{r}
\theta_{s_{1}}\left(\bar{x}_{s_{1}}\right)+\sum_{\left(s_{1}, u_{1}\right) \in \mathcal{E}_{f}} \theta_{s_{1} u_{1}}\left(\bar{x}_{s_{1}}, \bar{x}_{u_{1}}\right)+\theta_{s_{2}}\left(\bar{x}_{s_{2}}\right)+\sum_{\left(s_{2}, u_{2}\right) \in \mathcal{E}_{f}} \theta_{s_{2} u_{2}}\left(\bar{x}_{s_{2}}, \bar{x}_{u_{2}}\right)+ \\
+\theta_{s_{3}}\left(\bar{x}_{s_{3}}\right)+\sum_{\left(s_{3}, u_{3}\right) \in \mathcal{E}_{f}} \theta_{s_{3} u_{3}}\left(\bar{x}_{s_{3}}, \bar{x}_{u_{3}}\right)+\theta_{s_{4}}\left(\bar{x}_{s_{4}}\right)+\sum_{\left(s_{4}, u_{4}\right) \in \mathcal{E}_{f}} \theta_{s_{4} u_{4}}\left(\bar{x}_{s_{4}}, \bar{x}_{u_{4}}\right)= \\
\theta_{s}^{\prime}\left(x_{s}^{\prime}\right)+\sum_{(s, u) \in \mathcal{E}_{c}} \theta_{s u}^{\prime}\left(x_{s}^{\prime}, x_{u}^{\prime}\right)(12
\end{array}
$$

here $\mathcal{E}_{f}$ and $\mathcal{E}_{c}$ correspond to edge set in the fine and coarse scales, respectively. Considering the relative positions of the sites illustrated in Fig. 2 we have

$$
\begin{array}{r}
\bar{x}_{s_{1}}=\bar{x}_{s_{2}}=\bar{x}_{s_{3}}=\bar{x}_{s_{4}}=x_{s}^{\prime}, \\
\bar{x}_{s_{1} t}=x_{s t}^{\prime}, \bar{x}_{s_{1} r}=x_{s}^{\prime}, \bar{x}_{s_{1} b}=x_{s}^{\prime}, \bar{x}_{s_{1} l}=x_{s l}^{\prime}, \\
\bar{x}_{s_{2} t}=x_{s t}^{\prime}, \bar{x}_{s_{2} r}=x_{s r}^{\prime}, \bar{x}_{s_{2} b}=x_{s}^{\prime}, \bar{x}_{s_{2} l}=x_{s}^{\prime}, \\
\bar{x}_{s_{3} t}=x_{s}^{\prime}, \bar{x}_{s_{3} r}=x_{s r}^{\prime}, \bar{x}_{s_{3} b}=x_{s b}^{\prime}, \bar{x}_{s_{3} l}=x_{s}^{\prime}, \\
\bar{x}_{s_{4} t}=x_{s}^{\prime}, \bar{x}_{s_{4} r}=x_{s}^{\prime}, \bar{x}_{s_{4} b}=x_{s b}^{\prime}, \bar{x}_{s_{4} l}=x_{s l}^{\prime} .
\end{array}
$$

where we have used the subscripts $t, b, l, r$ to denote the top, bottom, left or the right neighbor of a site in an immediate four-connected neighborhood system. For the data term separately we can write

$$
\theta_{s}^{\prime}\left(x_{s}^{\prime}\right)=\theta_{s_{1}}\left(x_{s}^{\prime}\right)+\theta_{s_{2}}\left(x_{s}^{\prime}\right)+\theta_{s_{3}}\left(x_{s}^{\prime}\right)+\theta_{s_{4}}\left(x_{s}^{\prime}\right)=\sum_{i=1}^{4} \theta_{s_{i}}\left(x_{s}^{\prime}\right) .
$$

hence the data term associated with a block in the coarse level can be computed as the sum of its four corresponding nodes in the finer level which are 
themselves defined as the squared difference of magnitudes of the relevant elements on the normalized horizontal and vertical edge maps:

The pairwise potentials should satisfy:

$$
\begin{array}{r}
\theta_{s_{1}, s_{1} t}\left(\bar{x}_{s_{1}}, \bar{x}_{s_{1} t}\right)+\theta_{s_{1}, s_{1} r}\left(\bar{x}_{s_{1}}, \bar{x}_{s_{1} r}\right)+\theta_{s_{1}, s_{1} b}\left(\bar{x}_{s_{1}}, \bar{x}_{s_{1} b}\right) \\
+\theta_{s_{1}, s_{1} l}\left(\bar{x}_{s_{1}}, \bar{x}_{s_{1} l}\right)+\theta_{s_{2}, s_{2} t}\left(\bar{x}_{s_{2}}, \bar{x}_{s_{2} t}\right)+\theta_{s_{2}, s_{2} r}\left(\bar{x}_{s_{2}}, \bar{x}_{s_{2} r}\right) \\
+\theta_{s_{2}, s_{2} b}\left(\bar{x}_{s_{2}}, \bar{x}_{s_{2} b}\right)+\theta_{s_{2}, s_{2} l}\left(\bar{x}_{s_{2}}, \bar{x}_{s_{2} l}\right)+\theta_{s_{3}, s_{3} t}\left(\bar{x}_{s_{3}}, \bar{x}_{s_{3} t}\right) \\
+\theta_{s_{3}, s_{3} r}\left(\bar{x}_{s_{3}}, \bar{x}_{s_{3} r}\right)+\theta_{s_{3}, s_{3} b}\left(\bar{x}_{s_{3}}, \bar{x}_{s_{3} b}\right)+\theta_{s_{3}, s_{3} l}\left(\bar{x}_{s_{3}}, \bar{x}_{s_{3} l}\right) \\
+\theta_{s_{4}, s_{4} t}\left(\bar{x}_{s_{4}}, \bar{x}_{s_{4} t}\right)+\theta_{s_{4}, s_{4} r}\left(\bar{x}_{s_{4}}, \bar{x}_{s_{4} r}\right)+\theta_{s_{4}, s_{4} b}\left(\bar{x}_{s_{4}}, \bar{x}_{s_{4} b}\right) \\
+\theta_{s_{4}, s_{4} l}\left(\bar{x}_{s_{4}}, \bar{x}_{s_{4} l}\right)= \\
\theta_{s t}^{\prime}\left(x_{s}^{\prime}, x_{t}^{\prime}\right)+\theta_{s r}^{\prime}\left(x_{s}^{\prime}, x_{r}^{\prime}\right)+\theta_{s b}^{\prime}\left(x_{s}^{\prime}, x_{b}^{\prime}\right)+\theta_{s l}^{\prime}\left(x_{s}^{\prime}, x_{l}^{\prime}\right) .
\end{array}
$$

From (13) we have:

$$
\begin{array}{r}
\theta_{s_{1}, s_{1} t}\left(x_{s}^{\prime}, x_{t}^{\prime}\right)+\theta_{s_{1}, s_{1} l}\left(x_{s}^{\prime}, x_{l}^{\prime}\right)+\theta_{s_{2}, s_{2} t}\left(x_{s}^{\prime}, x_{t}^{\prime}\right)+ \\
\theta_{s_{2}, s_{2} r}\left(x_{s}^{\prime}, x_{r}^{\prime}\right)+\theta_{s_{3}, s_{3} r}\left(x_{s}^{\prime}, x_{r}^{\prime}\right)+\theta_{s_{3}, s_{3} b}\left(x_{s}^{\prime}, x_{b}^{\prime}\right)+ \\
\theta_{s_{4}, s_{4} b}\left(x_{s}^{\prime}, x_{b}^{\prime}\right)+\theta_{s_{4}, s_{4} l}\left(x_{s}^{\prime}, x_{l}^{\prime}\right)= \\
\theta_{s t}^{\prime}\left(x_{s}^{\prime}, x_{t}^{\prime}\right)+\theta_{s r}^{\prime}\left(x_{s}^{\prime}, x_{r}^{\prime}\right)+\theta_{s b}^{\prime}\left(x_{s}^{\prime}, x_{b}^{\prime}\right)+\theta_{s l}^{\prime}\left(x_{s}^{\prime}, x_{l}^{\prime}\right) .
\end{array}
$$

hence

$$
\begin{aligned}
\theta_{s t}^{\prime}\left(x_{s}^{\prime}, x_{t}^{\prime}\right) & =\theta_{s_{1}, s_{1} t}\left(x_{s}^{\prime}, x_{t}^{\prime}\right)+\theta_{s_{2}, s_{2} t}\left(x_{s}^{\prime}, x_{t}^{\prime}\right) \\
\theta_{s r}^{\prime}\left(x_{s}^{\prime}, x_{r}^{\prime}\right) & =\theta_{s_{2}, s_{2} r}\left(x_{s}^{\prime}, x_{r}^{\prime}\right)+\theta_{s_{3}, s_{3} r}\left(x_{s}^{\prime}, x_{r}^{\prime}\right) \\
\theta_{s b}^{\prime}\left(x_{s}^{\prime}, x_{b}^{\prime}\right) & =\theta_{s_{3}, s_{3} b}\left(x_{s}^{\prime}, x_{b}^{\prime}\right)+\theta_{s_{4}, s_{4} b}\left(x_{s}^{\prime}, x_{b}^{\prime}\right) \\
\theta_{s l}^{\prime}\left(x_{s}^{\prime}, x_{l}^{\prime}\right) & =\theta_{s_{1}, s_{1} l}\left(x_{s}^{\prime}, x_{l}^{\prime}\right)+\theta_{s_{4}, s_{4} l}\left(x_{s}^{\prime}, x_{l}^{\prime}\right)
\end{aligned}
$$

Adopting the quadratic pairwise potential

$$
\rho^{\prime}\left(x_{s}^{\prime}-x_{t}^{\prime}\right)^{2}=\rho\left(x_{s}^{\prime}-x_{t}^{\prime}\right)^{2}+\rho\left(x_{s}^{\prime}-x_{t}^{\prime}\right)^{2} .
$$

From equation (18) we find $\rho^{\prime}=2 \rho$, which means that the model prescribes a stronger interaction between sites in the higher levels of resolution. This is intuitive because the coarser resolutions sites represent larger groups of pixels which requires stronger interaction with each other. A comment regarding the multiresolution technique employed here is that the coarser scales considered here do not correspond to any coarser grids of the original image in a physical sense. The method is essentially a mathematical trick in which the model is changed so that its optimal solution maps to the optimal solution of the original problem, as discussed in [41]. 


\section{Statistical Shape Prior}

Deformable models can be broadly classified into two categories: free-form and parametric. In the free-form models (e.g. snake) only general continuity and smoothness constraints are considered [45]. As a result, these models can be matched to an arbitrary shape. In contrast, parametric models incorporate a general shape of the object of interest. They encode special attributes of an object and its variations and hence are more robust to occlusions and spurious structures (as G-Snake [46], Active Shape Model [47], [14] and [48]).

The pairwise potentials defined in [1] only impose a smoothness prior into the deformation. It is naturally expected that by injecting an object specific prior into the matching, better results can be obtained. This is even more important in the multiresolution approaches because one of the drawbacks of multiresolution approaches is that if an error occurs in a coarser scale of resolution, it will affect the solution in the finer scales. We constrain the solution in the coarsest scale using a shape prior so that the errors are minimal.

In order to construct a statistical shape model, we use frontal face images and match them to one another. Once a global spatial transformation (e.g. projective) is fitted to the set of corresponding points, the residual distortions are computed. The distribution of residual errors can then be approximated reasonably well by a Gaussian distribution [49]. In order to capture the main modes of the variation in the underlying distribution, the first $M$ eigenvectors of the covariance matrix are used as the basis vectors to construct a shape space. We consider the deformation in each direction separately, in the same way they are treated in the decomposed model. The distribution of the local deformations in each direction in the training set is expressed as

$$
p\left(X^{\prime \prime}\right)=\frac{\exp \left(-\frac{1}{2} \sum_{i=1}^{M} \frac{w_{i}^{2}}{e_{i}}\right)}{(2 \pi)^{M / 2} \prod_{k=1}^{M} e_{k}^{1 / 2}}
$$

where

$$
\begin{gathered}
\omega=\phi_{M}^{T}\left(X^{\prime}-\bar{X}_{\text {mean }}^{\prime}\right) \\
X^{\prime \prime}=\bar{X}_{\text {mean }}^{\prime}+\phi_{M} \omega
\end{gathered}
$$

is the vector of shape parameters determining the coordinates of the projected

point in the shape space with the elements, $w_{i} . X^{\prime}$ is the local deformation 
vector and $\bar{X}_{\text {mean }}^{\prime}$ is the mean deformation for the target class. $\phi_{M}$ is the matrix of the first $M$ principal eigenvectors of the covariance matrix of local deformations and $e_{i}, i=1 \ldots M$ denote the $M$ largest eigenvalues of the covariance matrix. Any local deformation $X^{\prime}$ in each direction can then be approximated by a linear combination of the $M$ eigenvectors corresponding to the largest eigenvalues as

$$
X^{\prime} \approx \bar{X}_{\text {mean }}^{\prime}+\phi_{M} \omega
$$

Projecting the local deformations into the shape space while retaining most of the variation compatible with the shape space, discards the variation of the deformation which is inconsistent with those reflected by the samples in the training set. The energy of the obtained local deformation in each direction projected into the shape space is denoted as

$$
E\left(X^{\prime}\right)=\frac{1}{2} \sum_{i=1}^{M} \frac{w_{i}^{2}}{e_{i}}
$$

\subsection{Regularizing and Constraining the Solution}

Having defined a statistical energy term for the local deformations, the new energy takes the form:

$$
E(X \mid \theta)=\sum_{s \in \mathcal{V}} \theta_{s}\left(x_{s}\right)+\sum_{(s, u) \in \mathcal{E}} \theta_{s u}\left(x_{s}, x_{u}\right)+\theta_{\text {prior }}(X)
$$

where

$$
\theta_{\text {prior }}(X)=\frac{1}{2} \sum_{i=1}^{M} \frac{w_{i x}^{2}}{e_{i x}}+\frac{1}{2} \sum_{i=1}^{M} \frac{w_{i y}^{2}}{e_{i y}}
$$

where $w_{i x}, w_{i y}, e_{i x}, e_{i y}$ denote shape parameters in the horizontal, shape parameters in the vertical direction and the first $M$ principal eigenvectors in the horizontal and vertical directions. We treat horizontal and vertical distortions separately (by having one eigenspace for each one) the same as in decomposed model, in which separate models are used for displacements in each direction. This is advantageous in terms of identification performance when severe head pose changes exist. It is apparent that the corresponding min-sum task incorporates cliques of size 1 (nodes), size 2 (ordinary edges) and a hyperedge containing all nodes (global interaction) represented by 
the data term, pairwise smoothness term and the local deformation energy term, respectively. The corresponding minimization problem needs two kinds of updates: between unary and binary constraints and between the unary and the global constraint. This kind of minimization has been addressed for example in [50] for a special type of global constraints in the case that each site has two admissible states. In our task, minimizing the energy in 24 incorporates min-marginalization of a function (shape prior energy) over the whole configuration space with the complexity exponential with the size of the full MRF model. Since the method would eventually be used in a recognition scenario, it needs to be extremely efficient. Instead of directly solving the problem in 24 , we propose a recursive two-stage approach:

- Solve the task without considering the prior energy term.

- Estimate the local deformations and project them into the shape space using (22). Add the global rigid transformation back to the local deformations and reduce the local neighborhood of each site in which correspondences are sought for the next round.

We repeat step one and two until the global shape parameters and the prior shape energy term do not change beyond a specified precision. In practice we found that it is not needed to repeat the two steps more than two times if the TRW-S method is iterated sufficiently. For pose estimation we use Levenberg-Marquardt [51] method. While first round of projection into the shape space makes the current solution compatible with those available in the training set, the next round refines the most up-to-date solution. In practice, as a result of reducing the searched neighborhood which effectively prunes the configurational space, the matching after the first iteration is computationally more efficient. The method is most similar to the eigen-snake model [52]. However, here we employ an efficient multi-scale optimization approach for the minimization of the energy function using the tree reweighted message passing method. Also, in the eigen-snake model the prior information is used to deform the template whereas we use it to constrain the solution and prune the configuration space. Using the proposed method for deformable registration, we obtain pixelwise correspondences between a pair of images. Figure 3 shows some examples. In the figure, the template, the target image, the deformed template and also the deformed template superimposed on the target image are illustrated for two pairs of images. 

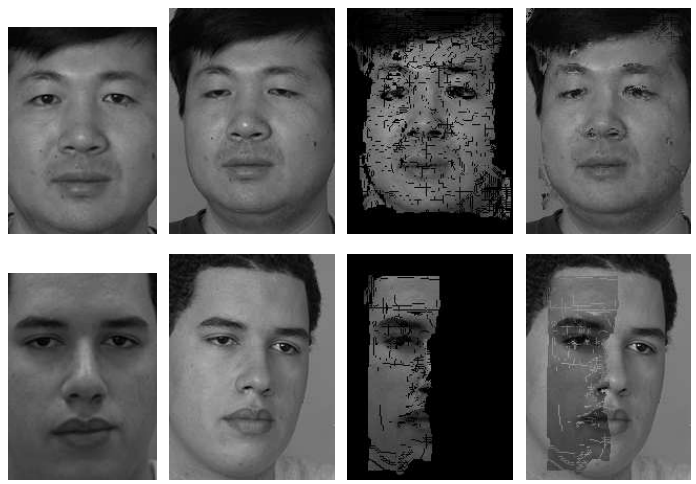

Figure 3: In each row from left to right: template, target, deformed template and deformed template superimposed on the target image.

\section{Classification}

Once the correspondences are established between a pair of images, in order to judge the goodness-of-match, we compute a similarity measure considering both textural and structural similarities between a pair of images. The structural similarity between the images, invariant to a global transformation, is best represented by the prior shape energy for the estimated match. While, the energy corresponding to the data term (considered as the difference between edge maps) can be used directly, we employ a more discriminative texture descriptor known as Local Binary Patterns.

\subsection{Textural Similarity}

Although we use facial images captured almost under constant illumination conditions, in order to remove any residual effects of uneven illumination conditions we apply a photometric normalization step before measuring the texture similarities. In [53] a photometric preprocessing method based on a series of steps is introduced. The method is designed to decrease the effects of changes in illumination conditions, highlights and local shadowing, while keeping the fundamental visual information. In the next step in order to extract features which are less affected by the unwanted illumination changes we use a local binary pattern operator [54]. The LBP operator is proved to be highly discriminative while being invariant to monotonic gray-level changes as well as computationally efficient. The original LBP operator assigns a label to every pixel of an image by comparing its $3 \times 3$-neighborhood with 
the value of the pixel under consideration and treating the results as a binary number. It is defined at a given pixel location $\left(x_{o}, y_{o}\right)$ as:

$$
\operatorname{LBP}\left(x_{o}, y_{o}\right)=\sum_{n=0}^{7} S\left(i_{n}-i_{o}\right) 2^{n}
$$

where $i_{o}$ is the gray value of the pixel under consideration and $i_{n}$ is the gray value of its 8 neighboring pixels. Function $S(x)$ is defined as:

$$
S(x)= \begin{cases}1 & x \geq 0 \\ 0 & x<0\end{cases}
$$

As long as the intensity order of the pixels in a neighborhood is preserved, LBPs are known to be unaffected by monotonic gray scale changes.

One extension to this operator proposes to use neighborhoods of different sizes in order to deal with textures at different resolutions [54]. The second extension to the original operator is the notion of uniform patterns. By considering the bit pattern circular, a local binary pattern is called uniform if the pattern has at most two bitwise transitions from 1 to 0 or vice versa. For face description in [54] the face image is divided into different subregions in order to extract local histograms. Once local histograms are extracted from each window, a spatially enhanced histogram is constructed by concatenating local histograms to form a global face descriptor. This histogram has three scales of description: in the lowest level are the LBP labels which include information about the patterns on the pixel-level, local histograms describe the image content in a regional level and finally the local histograms are concatenated to form a global description of the face image. While for the extraction of LBP histograms in [55] a regular division is used for both the gallery and test images, in the case of pose variation this approach is not very effective since different regions of the test image may correspond to different facial features, compared to the frontal gallery image.

Using the information available from image matching, for a window in the gallery image the corresponding region in the test image can be identified and the matched region in the test image can be used to extract LBP histogram. Extracted histograms from each region are normalized and concatenated into a single vector and compared using the $\chi^{2}$ distance:

$$
\chi^{2}(\eta, \xi)=\sum_{b, w} \frac{\left(\eta_{b, w}-\xi_{b, w}\right)^{2}}{\eta_{b, w}+\xi_{b, w}}
$$


where $\eta$ and $\xi$ are the normalized histograms of gallery and test images and $b$ and $w$ stand for the $b^{t h}$ bin of the histogram of the $w^{\text {th }}$ window in the images. For texture modeling, we use the Uniform LBP operator with radius 2 and use $(16 \times 16)$ windows in the gallery images and their corresponding patches in the probe images.

\subsection{Structural Similarity}

Different structures of faces can offer a discriminative measure for classification. Apparently this measure should be independent of the spatial transformation parameters of the probe image while at the same time should take into account the correlations of local deformations. These properties are met by the shape parameters discussed in Section 6. We use the prior energy term as a measure of structural similarity between a pair of facial images. Two observations about the imposition of the shape prior energy are as follows: because $w=0$ and consequently $\theta_{\text {prior }}=0$ when $X^{\prime}=\bar{X}_{\text {mean }}^{\prime}, w$ represent the local deformation parameters and as a result the rigid transformation parameters (e.g. projective) and the local deformation parameters are separated. Second, the imposition of the shape space constraint based on the prior knowledge of local deformations makes the matching more robust against spurious structures and outliers. The shape distance between a pair of images is defined as:

$$
D_{\text {Strcutural }}(I, J)=\sum_{i=1}^{M} \frac{w_{i x}^{2}}{e_{i x}}+\sum_{i=1}^{M} \frac{w_{i y}^{2}}{e_{i y}}
$$

Considering the definition of the energy function in (4), the overall energy is defined as the weighted sum of the data term (represented by ULBP histograms here) and the binary term (formulated in the PCA space). So it makes sense to combine these two terms after normalization in order to obtain the final distance measure as a weighted measure of shape and texture distances between a test image $(J)$ and $i^{\text {th }}$ gallery image $\left(I_{i}\right)$ :

$$
D\left(I_{i}, J\right)=\alpha \chi^{2}+(1-\alpha) D_{\text {Strcutural }}
$$

for $\alpha \in[0,1]$. D $D_{\text {Strcutural }}$ corresponds to structural distance and $\chi^{2}$ represents the textural differences of the images being compared. 


\section{Experimental evaluation}

In this section we provide the results of an experimental evaluation of the method in various scenarios. First, we compare the multi-scale matching method with the method in [1] in terms of their running time and the quality of the established match. Next, the proposed methodology for image matching is compared with that of [1] in a face identification scenario. The performance of the proposed face recognition algorithm is then compared with state-of-the-art algorithms on the challenging CMU-PIE database including a wide range of pose deviations. Finally, we provide the results of an identification test on the rotation shots of the FERET database for frontal and near frontal poses and compare the proposed approach to the state-ofthe-art methods for pose-invariant and also frontal face recognition.

Throughout our experiments, the coarsest scale we start the optimization in is constructed by nodes of size $4 \times 4$ pixels and the finest scale is $1 \times$ 1 , that is pixel level. Hence, the multiresolution analysis is performed in three scales. The range of displacements we search for correspondences is $[-36,+36]$ pixels in both horizontal and vertical directions. Gallery images we use in the experiments are normalized to the size of $160 \times 208$ in the CMU-PIE database and to the size $140 \times 196$ in the FERET database. In the experiments on CMU-PIE database where a severe head pose deviation exists in horizontal direction, only half of the face is used for matching and recognition. Deciding whether a severe pan movement exists in the image or not is made by comparing full vs. half face energies of the match.

\subsection{Computational Efficiency}

In order to compare the running times of the proposed multi-scale method with that of [1] fairly, we consider the method in [1] using pixelwise nodes instead of the block model. We let the two algorithms iterate till both reach an equal energy value. In Figure 4 the energy plots of the two algorithms are visualized per Log number of iterations. As it is evident from the plots, the multiresolution analysis achieves lower energy in fewer number of iterations. Of primary importance is that the computational complexity of one iteration for the method in [1] is much higher than that of the proposed multi-scale method. The efficiency in one iteration of the multi-scale scheme comes from two sources. First, the complexity of one message passing operation

in each scale of the multiresolution scheme is considerably less than that of the single-scale analysis due to the decimation of the disparity in coarser 


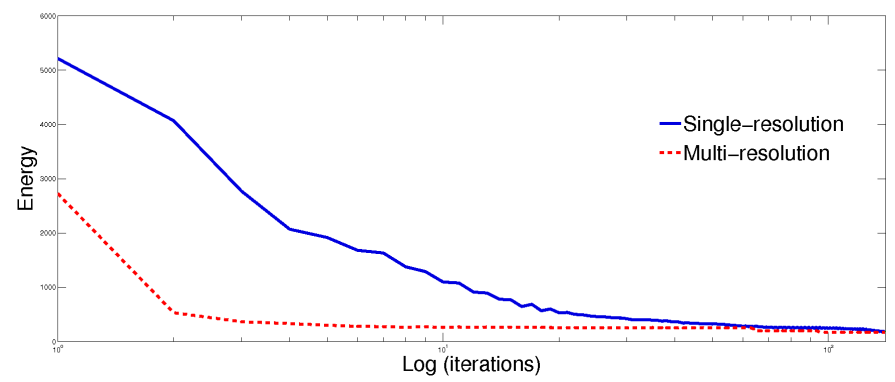

Figure 4: Comparison of multiresolution vs. single-resolution matching in terms of energy of the match.
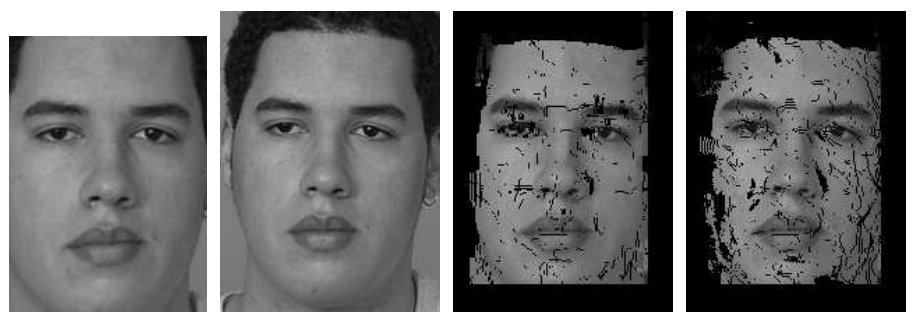

Figure 5: Comparison of multiresolution vs. single-resolution matching in terms of quality of the match. From left to right: template, target, multi-resolution result, single-resolution result

scales, and the constraints imposed by a coarser scale onto the next finer scale which effectively prunes out configurations that are not consistent with the previous coarse scale. Secondly, the number of nodes in all scales but the finest is lower than that of [1]. These two factors lead to a considerable speedup in matching by a factor of more than 5 .

Next, we visually compare the quality of match obtained by the two methods obtained by the two algorithms for an equal number of iterations. As before the method in [1] is constructed using pixelwise nodes rather than block model. Figure 5 illustrates a pair of images to be matched. In the same figure, the results of matching the template to the target image by warping the template image according to the found deformation are also shown. As can be seen from the figure, the multi-scale method leads to better results visually. This is due to the fact that in the multiscale analysis, the method becomes less prone to be affected by noise in images as a result of using groups of pixels as nodes in the coarser scales and also coarsening the configuration space which enables the method to take big jumps towards the minimum. 
Table 1: Comparison of the performance of the proposed matching method to the method in [1] in terms of Identification Rate.

\begin{tabular}{|c|c|c|c|}
\hline Pose & c05 & c07 & c22 \\
\hline \hline The method in [1] & 85 & 91 & 22 \\
\hline The proposed matching & 98 & 98 & 79 \\
\hline
\end{tabular}

\subsection{Performance Gains in Face Identification}

A question that might be asked is whether the proposed multi-scale analysis and shape prior constraint lead to any improvement in terms of the identification rate when compared to the method in [1]. In this section we show the benefits of using the proposed method in this work over that of [1]. We run the method in [1] using the block model. In order to compare the improvements over the method in [1], we keep texture and shape modeling the same for the two methods and just compare the two different matching methods. In our experiments we use a subset of the CMU-PIE database [56]. The subset is used to evaluate the performance of the system subject to pose changes, under almost constant illumination conditions with neutral expression. This subset, consists of 884 images of 68 subjects viewed from 13 different angles. Nine images of each subject are captured roughly at the head height, spanning a range of angles from approximately full right profile to full left profile. Two images are captured from the corners of the room while the other remaining two are captured from above and below the central camera. Figure 6 illustrates the pose variation present in this database. For our comparison, we use the images corresponding to frontal pose (pose 27) as gallery images and three different poses corresponding to full profile (pose 22 ), images with slight pose deviation in horizontal direction (pose 05), and images with slight pose deviation in vertical direction (pose 07) as test images. The performance of the proposed method with that of [1] is reported in Table 1. The method constantly outperforms the one in [1] in terms of identification performance. As evident from the results, the proposed approach can be particularly beneficial where there are severe head pose changes (e.g. pose 22).

\subsection{Comparison with Other Face Recognition Algorithms}

In this section we compare the performance of the proposed approach with some other state-of-the-art methods for face recognition on the CMUPIE database. In this experiment, as in the previous section we use frontal 


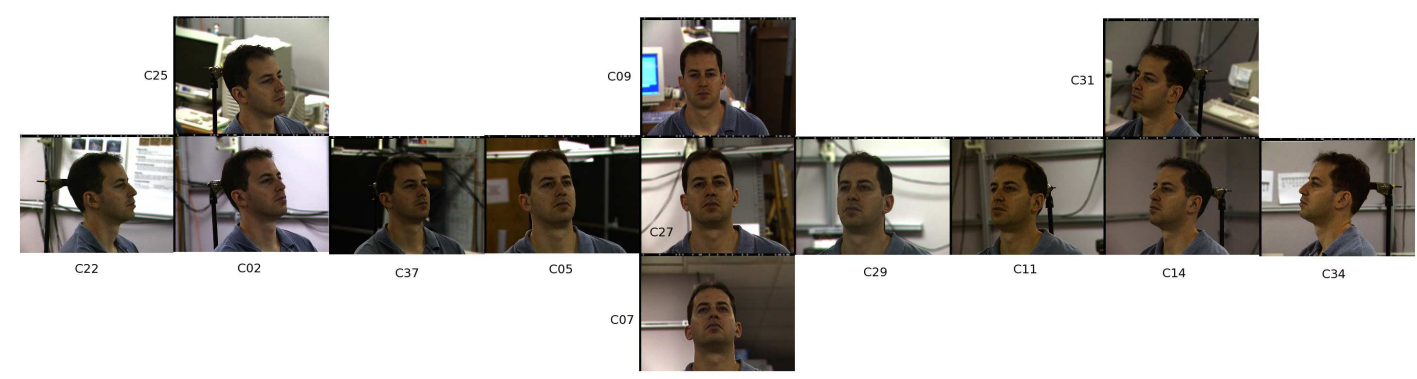

Figure 6: Illustration of pose variation in CMU-PIE dataset.

views of subjects (pose 27) as gallery images while all the other poses are considered as probe images.

In Table 2 we present the results and compare them to some other approaches. The reported results for the other approaches are taken from the literature. We also provide relevant details about the approaches in the Table 3. In these methods we have provided the identification rates reported using frontal images (pose C27) as gallery images. It can be observed that the proposed technique achieves comparable or sometimes better performance to most of the other approaches with less restrictive assumptions. In the comparison one needs to take into account the following considerations. The best performing method in Table 2 [16] uses non-frontal gallery images as well as frontal images for training, whereas we do not use any non-frontal training images. Also, the method in [16] uses 80 manually labeled landmark points, whereas the method proposed here does not need any landmark points and only requires the face to be detected in a bounding box which is much easier than providing landmarks automatically. Other advantages of the method proposed here are that it can also cope with moderate global spatial transformation (e.g. projective) between the images.

The performance of the proposed approach for matching in terms of face identification on the CMU-PIE database is also compared with another registration method [57]. The method in [57] proposes a face recognition pipeline composed of face detection, landmark localization, feature extraction and identification. As we want to compare the performance of the registration method in [57], we feed the two system with the same face images, bypassing the detection method in [57]. For this experiment we use the online code provided by the authors. We use the landmarks obtained by running the method in [57] to normalize the image using an affine transformation. The 
Table 2: Comparison of the performance of the proposed approach to the state-of-the-art methods on the CMU-PIE database.

\begin{tabular}{|c|c|c|c|c|c|c|c|c|c|c|c|c|}
\hline Pose & $\mathrm{CO2}$ & $\mathrm{CO5}$ & $\mathrm{Co7}$ & $\mathrm{Co9}$ & C11 & $\mathrm{C14}$ & $\mathrm{C22}$ & $\mathrm{C25}$ & $\mathrm{C29}$ & C31 & C34 & C37 \\
\hline Pan deviation & $-44^{\circ}$ & $-16^{\circ}$ & $0^{\circ}$ & $0^{\circ}$ & $32^{\circ}$ & $47^{\circ}$ & $-62^{\circ}$ & $-44^{\circ}$ & $17^{\circ}$ & $47^{\circ}$ & $66^{\circ}$ & $-31^{\circ}$ \\
\hline Tilt deviation & $0^{\circ}$ & $0^{\circ}$ & $-13^{\circ}$ & $13^{\circ}$ & $0^{\circ}$ & $0^{\circ}$ & $1^{\circ}$ & $11^{\circ}$ & $0^{\circ}$ & $11^{\circ}$ & $1^{\circ}$ & $0^{\circ}$ \\
\hline L-fields [18] & 58 & 94 & 89 & 94 & 88 & 70 & 38 & 56 & 57 & 56 & 47 & 89 \\
\hline PDM [13] & 72 & 100 & na & na & 94 & 62 & na & na & 98 & na & 20 & 97 \\
\hline AA-LBP [16] & 95 & 100 & 100 & 100 & 100 & 91 & na & 89 & 100 & 80 & 73 & 100 \\
\hline 3D model [22] & 76 & 99 & 99 & 99 & 93 & 87 & 50 & 75 & 97 & 78 & 49 & 94 \\
\hline Norm. [57] & 11 & 51 & 48 & 54 & 26 & 8 & 3 & 8 & 51 & 13 & 4 & 22 \\
\hline This work & 95 & 98 & 98 & 100 & 89 & 91 & 79 & 95 & 91 & 88 & 83 & 100 \\
\hline
\end{tabular}

Table 3: Some specifications of the methods in Table 2 and test details.

\begin{tabular}{|c|c|c|c|}
\hline Method & Non-frontal training image & no. of landmark points used & no. of subjects used \\
\hline \hline L-fields [18] & $\mathbf{Y}$ & 39-54 depending on pose & $\mathbf{3 4}$ \\
\hline PDM [13] & $\mathbf{Y}$ & $\mathbf{6 2}$ & $\mathbf{6 8}$ \\
\hline AA-LBP [16] & $\mathbf{Y}$ & $\mathbf{8 0}$ & $\mathbf{6 8}$ \\
\hline 3D model [22] & 3D data & $>6$ & $\mathbf{6 8}$ \\
\hline This work & $\mathbf{N}$ & None & $\mathbf{6 8}$ \\
\hline
\end{tabular}

rest of the procedure is kept the same as the procedure advocated in this work. The results obtained are reported in Table 2 referred to as Norm.. One observes that the proposed approach can outperform the method in [57] by a large margin. This can be affiliated to a number of reasons. First, the method in [57] fails for large pose deviations from frontal. This leads to a large error in geometric normalization. Second, even in near frontal poses when the method in [57] gives reasonable results, it is outperformed by the method in this work, this is because our method establishes dense correspondences which leads to accurate comparison of the two faces in terms of their shape and texture. It conclusion the proposed approach is especially beneficial when only one gallery is available per subject and test data includes images with large deviation from frontal.

\subsection{Identification Test on the FERET Database}

In this experiment we investigate the applicability of the proposed approach for recognition of frontal and near frontal face images on a larger database and compare our results with the state-of-the-art methods. For this experiment we use images which have been captured at viewpoints $b f$, $b a$ and $b e$ of FERET database which roughly correspond to $-15^{\circ}, 0^{\circ},+15^{\circ}$. As stated earlier, we use eye coordinates to crop frontal gallery images but 
Table 4: Comparison of the performance of the proposed approach to the state-of-the-art methods on FERET database.

\begin{tabular}{|c|c|c|c|c|}
\hline Pose & Kanade and Yamda [26] & Online Flow $[27]$ & Stack Flow [27] & This work [27] \\
\hline bf & 85 & 80 & 90 & 88.5 \\
\hline be & 80 & 70 & 82 & 89 \\
\hline
\end{tabular}

for non-frontal images we use the face detection method in [6] to detect the face and enclose it in a bounding box. The results of the comparison with two other approaches on poses be, bf are provided in Table 4.

It is worth noting that both methods in [27] and [26] use eye coordinates to crop the face out of the image whereas we use a face detector. Also, we used 200 subjects in the test and do not use non-frontal images in training whereas other methods use 100 subjects and use non-frontal images for training. In spite of the aforementioned factors, it can be observed that the proposed method outperforms other approaches.

In another experiment we compare the performance of the system with a state-of-the-art method developed for frontal images [58]. The method in [58] achieves $99.5 \%$ recognitions rate for the frontal pose $b a$ and the performance of the proposed approach is $97 \%$ which is not far from the optimal performance. The results for the two near frontal poses are as in Table 5. For these poses, we report the results obtained using the method in [58] in two different situations. The first row in Table 5 reports the results using manually annotated eye coordinates. The second row of Table 5 reports the results obtained by the method in [58] without using the eye coordinates but using the face detection data as used in the proposed method. From the table it can be observed that in a more realistic situation where detection of eye coordinates becomes difficult (as a result of pose or expression change) while the performance of the state-of-the-art method in [58] drops significantly, the proposed approach performs reasonably well. The reason why the proposed approach does not outperform the method in [58] when eye coordinates are used for the [58], is that we use only one gallery image for texture comparison whereas in [58] the method uses large training data for multiresolution LBP feature representation.

Throughout our experimental evaluations, we used images captured almost under constant illumination conditions. However, in the presence of extreme lighting changes resulting in shadowing effects which produce artificial edges in the images, the data term used in this model (constructed using edge magnitudes) will be sensitive to noise which could degrade the match 
Table 5: Comparison of the performance of the proposed approach to the state-of-the-art methods on the FERET database.

\begin{tabular}{|c|c|c|}
\hline Pose & bf & be \\
\hline \hline The method in [58] using manually labeled eye coordinates & 93 & 92 \\
\hline The method in [58] using face detection data & 54.5 & 56 \\
\hline This work using face detection data & 88.5 & 89 \\
\hline
\end{tabular}

quality and subsequently the performance. In order to remedy this effect one may use effective illumination normalization methods before matching or employ near infra-red images which are believed to be less affected by illumination changes.

\section{Conclusion}

The problem of pose-invariant face recognition is addressed. We proposed an MRF classification system which employs the energy of the established match between a pair of images as a criterion for classification. The system is robust to moderate global spatial transformations. A number of enhancements over the image matching method in [1] are proposed. First, based on super-coupling transform, a multi-scale relaxation scheme using sequential tree re-weighted message passing approach [2] was proposed to speed-up the matching process and to increase its invariance to noise. Next, by taking advantage of a statistical shape prior, the matching process was regularized and constrained which made it robust to spurious structures and outliers. In the classification, both textural and structural similarities of the facial images have been taken into account. The method achieved good performance on two face databases both on frontal and out-of-plain rotated face images.

\section{References}

[1] A. Shekhovtsov, I. Kovtun, V. Hlavac, Efficient mrf deformation model for non-rigid image matching, Computer Vision and Image Understanding 112 (1) (2008) 91 - 99, special Issue on Discrete Optimization in Computer Vision.

[2] V. Kolmogorov, Convergent tree-reweighted message passing for energy minimization, Pattern Analysis and Machine Intelligence, IEEE Transactions on 28 (10) (2006) 1568-1583. 
[3] W. Zhao, R. Chellappa, A. Rosenfeld, P. J. Phillips, Face recognition: A literature survey, ACM Computing Surveys (2003) 399-458.

[4] A. Ahmadyfard, J. Kittler, The role of relational constraints in region matching, International Journal of Pattern Recognition and Artificial Intelligence 18 (2004) 443-454.

[5] S. R. Arashloo, J. Kittler, Pose-invariant face matching using mrf energy minimization framework, in: EMMCVPR, 2009, pp. 56-69.

[6] Z. Kalal, J. Matas, K. Mikolajczyk, Weighted sampling for large-scale boosting, in: British Machine Vision Conference, 2008.

[7] A. Pentland, B. Moghaddam, T. Starner, View-based and modular eigenspaces for face recognition, in: Computer Vision and Pattern Recognition, 1994. Proceedings CVPR '94., 1994 IEEE Computer Society Conference on, 1994, pp. 84-91.

[8] D. Beymer, Face recognition under varying pose, in: Computer Vision and Pattern Recognition, 1994. Proceedings CVPR '94., 1994 IEEE Computer Society Conference on, 1994, pp. 756-761.

[9] R. Singh, M. Vatsa, A. Ross, A. Noore, A mosaicing scheme for poseinvariant face recognition, Systems, Man, and Cybernetics, Part B: Cybernetics, IEEE Transactions on 37 (5) (2007) 1212-1225.

[10] M. Vasilescu, D. Terzopoulos, Multilinear image analysis for facial recognition, in: Pattern Recognition, 2002. Proceedings. 16th International Conference on, Vol. 2, 2002, pp. 511-514 vol.2.

[11] A. Georghiades, P. Belhumeur, D. Kriegman, From few to many: illumination cone models for face recognition under variable lighting and pose, Pattern Analysis and Machine Intelligence, IEEE Transactions on 23 (6) (2001) 643-660.

[12] D. Beymer, T. Poggio, Face recognition from one example view, in: Computer Vision, 1995. Proceedings., Fifth International Conference on, 1995, pp. 500-507. 
[13] D. Gonzalez-Jimenez, J. Alba-Castro, Toward pose-invariant 2-d face recognition through point distribution models and facial symmetry, Information Forensics and Security, IEEE Transactions on 2 (3) (2007) 413-429.

[14] T. Cootes, G. Edwards, C. Taylor, Active appearance models, Pattern Analysis and Machine Intelligence, IEEE Transactions on 23 (6) (2001) 681-685.

[15] T. Cootes, K. Walker, C. Taylor, View-based active appearance models, in: Automatic Face and Gesture Recognition, 2000. Proceedings. Fourth IEEE International Conference on, 2000, pp. 227-232.

[16] X. Zhang, Y. Gao, M. Leung, Recognizing rotated faces from frontal and side views: An approach toward effective use of mugshot databases, Information Forensics and Security, IEEE Transactions on 3 (4) (2008) 684-697.

[17] S. Prince, J. Warrell, J. Elder, F. Felisberti, Tied factor analysis for face recognition across large pose differences, Pattern Analysis and Machine Intelligence, IEEE Transactions on 30 (6) (2008) 970-984.

[18] R. Gross, I. Matthews, S. Baker, Appearance-based face recognition and light-fields, Pattern Analysis and Machine Intelligence, IEEE Transactions on 26 (4) (2004) 449-465.

[19] V. Blanz, S. Romdhani, T. Vetter, Face identification across different poses and illuminations with a $3 \mathrm{~d}$ morphable model, in: Automatic Face and Gesture Recognition, 2002. Proceedings. Fifth IEEE International Conference on, 2002, pp. 192-197.

[20] V. Blanz, S. Romdhani, T. Vetter, Face identification across different poses and illuminations with a $3 \mathrm{~d}$ morphable model, in: Automatic Face and Gesture Recognition, 2002. Proceedings. Fifth IEEE International Conference on, 2002, pp. 192-197.

[21] X. Liu, T. Chen, Pose-robust face recognition using geometry assisted probabilistic modeling, in: Computer Vision and Pattern Recognition, 2005. CVPR 2005. IEEE Computer Society Conference on, Vol. 1, 2005, pp. 502-509 vol. 1. 
[22] S. Romdhani, V. Blanz, T. Vetter, Face identification by fitting a 3d morphable model using linear shape and texture error functions, in: ECCV '02: Proceedings of the 7th European Conference on Computer Vision-Part IV, Springer-Verlag, London, UK, 2002, pp. 3-19.

[23] J. Tena, R. Smith, M. Hamouz, J. Kittler, A. Hilton, J. Illingworth, 2d face pose normalisation using a 3d morphable model, in: Proceedings of the International Conference on Video and Signal Based Surveillance, 2007, pp. 1-6.

[24] T.-K. Kim, J. Kittler, Locally linear discriminant analysis for multimodally distributed classes for face recognition with a single model image, Pattern Analysis and Machine Intelligence, IEEE Transactions on 27 (3) (2005) 318-327.

[25] J. Huang, P. Yuen, W.-S. Chen, J. H. Lai, Choosing parameters of kernel subspace lda for recognition of face images under pose and illumination variations, Systems, Man, and Cybernetics, Part B: Cybernetics, IEEE Transactions on 37 (4) (2007) 847-862.

[26] T. Kanade, A. Yamada, Multi-subregion-based probabilistic approach toward pose-invariant face recognition, in: Computational Intelligence in Robotics and Automation, 2003. Proceedings. 2003 IEEE International Symposium on, Vol. 2, 2003, pp. 954-959 vol.2.

[27] A. Ashraf, S. Lucey, T. Chen, Learning patch correspondences for improved viewpoint invariant face recognition, in: Computer Vision and Pattern Recognition, 2008. CVPR 2008. IEEE Conference on, 2008, pp. $1-8$.

[28] D. Graham, N. Allinson, Face recognition from unfamiliar views: subspace methods and pose dependency, in: Automatic Face and Gesture Recognition, 1998. Proceedings. Third IEEE International Conference on, 1998, pp. 348-353.

[29] C. Castillo, D. Jacobs, Using stereo matching for 2-d face recognition across pose, in: Computer Vision and Pattern Recognition, 2007. CVPR '07. IEEE Conference on, 2007, pp. 1-8.

[30] R. Wang, Z. Lei, M. Ao, S. Z. Li, Bayesian face recognition based on markov random field modeling, in: ICB '09: Proceedings of the Third 
International Conference on Advances in Biometrics, Springer-Verlag, Berlin, Heidelberg, 2009, pp. 42-51.

[31] B.-G. Park, K.-M. Lee, S.-U. Lee, Face recognition using face-arg matching, Pattern Analysis and Machine Intelligence, IEEE Transactions on 27 (12) (2005) 1982-1988.

[32] R. Huang, V. Pavlovic, D. Metaxas, A hybrid face recognition method using markov random fields, in: Pattern Recognition, 2004. ICPR 2004. Proceedings of the 17th International Conference on, Vol. 3, 2004, pp. 157-160 Vol.3.

[33] J. Zhu, L. V. Gool, S. C. Hoi, Unsupervised face alignment by robust nonrigid mapping, in: Computer Vision, 2009 IEEE 12th International Conference on, 2009, pp. $1265-1272$.

[34] P. Li, S. Prince, Joint and implicit registration for face recognition, in: Computer Vision and Pattern Recognition, 2009. CVPR 2009. IEEE Conference on, 2009, pp. $1510-1517$.

[35] G. Huang, V. Jain, E. Learned-Miller, Unsupervised joint alignment of complex images, in: Computer Vision, 2007. ICCV 2007. IEEE 11th International Conference on, 2007, pp. $1-8$. doi:10.1109/ICCV.2007.4408858.

[36] T. Werner, A linear programming approach to max-sum problem: A review, Pattern Analysis and Machine Intelligence, IEEE Transactions on 29 (7) (2007) 1165-1179.

[37] S. Aji, R. McEliece, The generalized distributive law, Information Theory, IEEE Transactions on 46 (2) (2000) 325-343.

[38] R. Szeliski, R. Zabih, D. Scharstein, O. Veksler, V. Kolmogorov, A. Agarwala, M. Tappen, C. Rother, A comparative study of energy minimization methods for markov random fields with smoothness-based priors, Pattern Analysis and Machine Intelligence, IEEE Transactions on 30 (6) (2008) 1068-1080.

[39] P. F. Felzenszwalb, D. P. Huttenlocher, J. M. Kleinberg, Fast algorithms for large-state-space hmms with applications to web usage analysis, in: In Advances in Neural Information Processing Systems, 2003, p. 2003. 
[40] B. Gidas, A renormalization group approach to image processing problems, Pattern Analysis and Machine Intelligence, IEEE Transactions on 11 (2) (1989) 164-180.

[41] M. Petrou, Accelerated optimization via the renormalization group transform, Complex Stochastic Systems and Engineering.London: Clarendon Press (1993) 105-120.

[42] M. Bober, M. Petrou, J. Kittler, Nonlinear motion estimation using the supercoupling approach, Pattern Analysis and Machine Intelligence, IEEE Transactions on 20 (5) (1998) 550-555.

[43] P. Felzenszwalb, D. Huttenlocher, Efficient belief propagation for early vision, in: Computer Vision and Pattern Recognition, 2004. CVPR 2004. Proceedings of the 2004 IEEE Computer Society Conference on, Vol. 1, 2004, pp. I-261-I-268 Vol.1.

[44] P. Tipwai, S. Madarasmi, A coarse-and-fine bayesian belief propagation for correspondence problems in computer vision, in: MICAI, 2007, pp. 683-693.

[45] A. Singh, D. Terzopoulos, D. B. Goldgof, Deformable Models in Medical Image Analysis, IEEE Computer Society Press, Los Alamitos, CA, USA, 1998.

[46] K. Lai, R. Chin, Deformable contours: modeling and extraction, Pattern Analysis and Machine Intelligence, IEEE Transactions on 17 (11) (1995) 1084-1090.

[47] T. F. Cootes, C. J. Taylor, D. H. Cooper, J. Graham, Active shape models-their training and application, Computer Vision and Image Understanding 61 (1) (1995) 38-59.

[48] A. L. Yuille, D. S. Cohen, P. W. Hallinan, Feature extraction from faces using deformable templates, in: Computer Vision and Pattern Recognition, 1989. Proceedings CVPR '89., IEEE Computer Society Conference on, 1989, pp. 104-109.

[49] B. Moghaddam, A. Pentland, Probabilistic visual learning for object representation, Pattern Analysis and Machine Intelligence, IEEE Transactions on 19 (7) (1997) 696-710. 
[50] T. Werner, High-arity interactions, polyhedral relaxations, and cutting plane algorithm for soft constraint optimisation (map-mrf), in: Computer Vision and Pattern Recognition, 2008. CVPR 2008. IEEE Conference on, 2008, pp. 1-8.

[51] W. H. Press, B. P. Flannery, S. A. Teukolsky, W. T. Vetterling, Numerical Recipes in C: The Art of Scientific Computing, 2nd Edition, Cambridge University Press, 1992.

[52] Z. Xue, S. Z. Li, E. K. Teoh, AI-EigenSnake: an affine-invariant deformable contour model for object matching, Image and Vision Computing 20 (2) (2002) 77-84.

[53] X. Tan, B. Triggs, Enhanced local texture feature sets for face recognition under difficult lighting conditions, in: AMFG, 2007, pp. 168-182.

[54] T. Ojala, M. Pietikainen, T. Maenpaa, Multiresolution gray-scale and rotation invariant texture classification with local binary patterns, Pattern Analysis and Machine Intelligence, IEEE Transactions on 24 (7) (2002) 971-987.

[55] T. Ahonen, A. Hadid, M. Pietikainen, Face description with local binary patterns: Application to face recognition, Pattern Analysis and Machine Intelligence, IEEE Transactions on 28 (12) (2006) 2037-2041.

[56] T. Sim, S. Baker, M. Bsat, The cmu pose, illumination, and expression (pie) database, in: Automatic Face and Gesture Recognition, 2002. Proceedings. Fifth IEEE International Conference on, 2002, pp. 46-51.

[57] M. Everingham, J. Sivic, A. Zisserman, Hello! my name is... buffy automatic naming of characters in tv video, in: In BMVC, 2006.

[58] C.-H. Chan, J. Kittler, K. Messer, Multi-scale local binary pattern histograms for face recognition, in: Proceedings of International Conference on Biometrics, Springer, 2007, pp. 809-818. 